\title{
Numerical and Experimental Investigation of an Ultrawideband Ridged Pyramidal Horn Antenna With Curved Launching Plane for Pulse Radiation
}

\author{
Xu Li, Student Member, IEEE, Susan C. Hagness, Member, IEEE, Min K. Choi, Student Member, IEEE, and \\ Daniel W. van der Weide, Member, IEEE
}

\begin{abstract}
We report the numerical analysis and experimental characterization of an ultrawideband (UWB) antenna designed for radiating short microwave pulses. The antenna consists of a pyramidal horn, a ridge, and a curved launching plane terminated with resistors. Detailed three-dimensional finite-difference time-domain (FDTD) simulations have been conducted to assist with the characterization of the antenna. FDTD results are compared with experimental data and are shown to be in good agreement. We demonstrate that the antenna exhibits a very low voltage standing wave ratio $(\leq 1.5)$ over a wide frequency range from 1 to $11 \mathrm{GHz}$ and a very high fidelity $(\geq 0.92)$. The spatial distribution of radiated energy is characterized both in the time domain, using transient field observations at various angles, as well as in the frequency domain, using single-frequency far-field radiation patterns. We conclude that this antenna offers high-fidelity transmission and reception of ultrashort microwave pulses with minimal distortion.
\end{abstract}

Index Terms-Breast cancer detection, finite-difference time-domain (FDTD), horn antennas, impulse radar, microwave imaging, ultrawideband (UWB) antennas.

\section{INTRODUCTION}

$\mathbf{R}$ ECENT research on ultrawideband (UWB) antennas has been motivated in part by a growing interest in the use of short-pulse radar techniques in remote and subsurface sensing applications at radio, microwave, and terahertz frequencies. Compared to narrowband systems, UWB radar offers potentially higher imaging resolution and better target characterization. However, the design and fabrication of high-performance transmitting/receiving antennas often presents significant challenges in the implementation of these systems. Typical antenna design requirements include a broad operating bandwidth for short pulse radiation, a compact radiation pattern to avoid interference caused by backscatter from undesired directions and a small size to make the system portable.

Manuscript received July 18, 2003; revised September 15, 2003. This work was supported by the Department of Defense Breast Cancer Research Program under award DAMD17-02-1-0625, by the National Science Foundation under Grant BES-0201880, by the National Institutes of Health under Grant R21 CA92188-01 awarded by the National Cancer Institute, by ARO under Grant DAAD19-02-1-0081, and by AFOSR and DARPA under Grant F49620-02-1-0329.

The authors are with the Department of Electrical and Computer Engineering, University of Wisconsin, Madison, WI 53706 USA (e-mail: xuli@students.wisc.edu; hagness@engr.wisc.edu; mchoi@cae.wisc.edu; danvdw@engr.wisc.edu).

Digital Object Identifier 10.1109/LAWP.2003.820708
Our investigation of pulse-radiating antennas has been primarily motivated by our ongoing work on space-time microwave imaging for breast cancer detection [1]. In this approach, each antenna in an array sequentially transmits a low-power UWB microwave pulse into the breast. Robust space-time beamforming algorithms are applied to the received backscatter signals to create an image of backscattered energy as a function of location. The use of UWB pulses combined with the spatial focusing capabilities of the beamformer has been shown to be effective in discriminating against clutter generated by heterogeneity of normal breast tissue. The operating frequency range of this imaging system is approximately 1-11 GHz. This range was chosen to ensure both a reasonably high imaging resolution and sufficient depth of penetration. Accordingly, we seek UWB antennas with centimeter-scale dimensions for use in this medical sensing application.

Typical examples of wideband antennas used for pulse radiation include resistively loaded monopoles, dipoles, bow-tie antennas, and various forms of horn antennas [2]-[6]. It has been noted that the bandwidth of horn antennas can be increased significantly by adding metallic ridges to the waveguide and flared sections [7]. Numerical and experimental investigations of pyramidal horn antennas with double ridges have been reported previously [8]. This type of antenna is commercially available with an operational frequency band of 1-18 GHz. However, the large dimensions and high cost of these antennas make them unsuitable for medical imaging applications.

In this letter, we investigate a modified version of the ridged horn antenna in which the waveguide section is eliminated and one of the two ridges is replaced by a curved metallic plane terminated by resistors. The generic form of this configuration has been proposed in [9]. To the best of our knowledge, however, a detailed numerical analysis and experimental characterization of this type of antenna has not been reported in the literature. In this letter, we present a numerical and experimental study of a specific realization of this design, wherein the antenna is customized to centimeter-scale dimensions for operation in the microwave frequency range from 1 to $11 \mathrm{GHz}$.

\section{Description of the ANTENNA GEOMETRY}

Fig. 1 illustrates the antenna geometry. The antenna consists of a pyramidal horn radiation cavity, a metallic ridge, and a curved metallic launching plane terminated with resistors. 


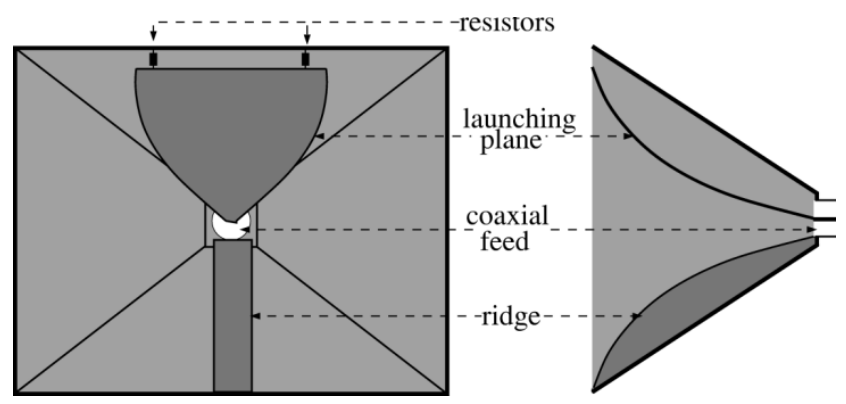

(a)

(b)

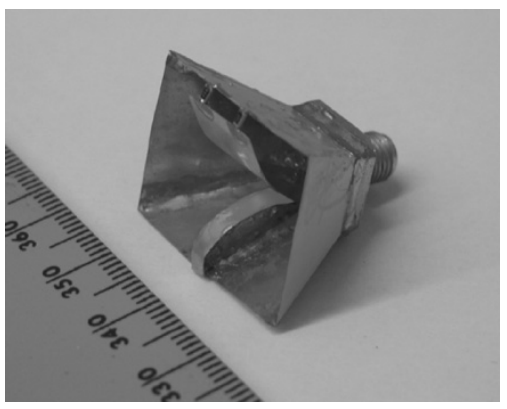

(c)

Fig. 1. Geometry of the antenna. (a) End view. (b) Side view. (c) Photo of the fabricated antenna. The adjacent ruler is marked in units of centimeters.

The pyramidal horn is connected to the outer conductor of the coaxial feed and serves as the ground plane, providing a current return path. Because of the coaxial feed, this ground-plane configuration eliminates the need for an UWB balun. The ground plane also confines the main beam of the radiation pattern to the opening of the horn, ensuring a compact radiation pattern.

The launching plane is a curved planar structure connected to the central conductor of the coaxial feed. As shown in Fig. 1(a) and (b), the launching plane curves toward one of the side walls of the pyramidal horn and tapers toward the feed point. Termination resistors are attached between the end of the launching plane and the side wall of the pyramidal horn. Microwave energy is directed and launched by this curved plane into the surrounding medium. The termination resistors suppress reflections from the end of the launching plane. Fig. 1(a) and (b) also shows the shape of the ridge which is attached to the side wall opposite the curved launching plane. The top surface of the ridge curves toward the antenna aperture.

The dimensions of the horn are chosen based on the physical size requirements and the operating frequency range associated with the desired application. The curvature and shape of the launching plane, the thickness and the contour of the curved side of the ridge, and the termination resistors are the main factors influencing the input impedance of the antenna. These parameters are chosen to minimize reflections at the feed point as well as at any point along the antenna. In order to match the $50-\Omega$ input impedance of the feeding coaxial cable, two $100-\Omega$ termination resistors are connected in parallel near opposite corners of the launching plane [see Fig. 1(a) and (c)]. Chip resistors are used to minimize inductance. Other geometrical parameters of the antenna are optimized using simulations and experimental measurements. In the finished design, the pyramidal horn has a depth of $13 \mathrm{~mm}$ with a $25 \mathrm{~mm} \times 20 \mathrm{~mm}$ aperture. The maximum width of the launching plane is $12 \mathrm{~mm}$, and the thickness of the ridge is $2 \mathrm{~mm}$. The antenna is fabricated using brass sheets and connected to a coaxial cable via an SMA connector. The individual components of the antenna are joined by soldering.

\section{NUMERICAL AND EXPERIMENTAL CHARACTERIZATION}

We have developed a three-dimensional (3-D) finite-difference time-domain (FDTD) model of the antenna to assist with the characterization and optimization of the antenna performance. The antenna geometry is modeled using a uniform space lattice with a $0.5-\mathrm{mm}$ grid resolution. The curved or flared metal surfaces of the antenna are modeled using a staircased perfectly electrical conducting (PEC) approximation. The base of the pyramidal horn is covered by a small PEC plane to provide a complete ground plane. A vertical gap of one grid cell exists between the feed point of the launching plane and the base of the horn. The antenna excitation is implemented using a $1.0-\mathrm{V}, 50-\Omega$ resistive voltage source across the gap [10]. The 100- $\Omega$ termination resistors attached to the end of the launching plane are also incorporated into the FDTD model as lumped circuit elements [10]. The FDTD grid is terminated with a Berenger perfectly matched layer (PML) absorbing boundary condition [11].

Data for our experimental characterization of the antenna is acquired using an Agilent E8364A performance network analyzer (PNA). All time-domain "measured" waveforms discussed below have been generated synthetically using the PNA in a swept frequency mode. Measurements are conducted from 1 to $11 \mathrm{GHz}$ using 201 frequency samples. Frequency-domain data is scaled by the spectrum of the desired UWB input pulse and converted to the time domain using an inverse discrete Fourier transform (DFT).

The UWB performance of the antenna can be characterized in the frequency domain by the input reflection coefficient (which, in this case, is equal to $S_{11}$ ) or the voltage standing wave ratio (VSWR). In our experiments, $S_{11}$ is recorded and converted to VSWR. In our FDTD simulations, a source voltage waveform with spectral content covering the desired frequency range is excited at the feed point. Current and voltage waveforms $v(t)$ and $i(t)$ are recorded at the source location in the grid and converted to a frequency-domain input voltage $\left(V_{\text {in }}\right)$ and current $\left(I_{\text {in }}\right)$ using a forward DFT. Then, the input impedance, $Z_{\text {in }}$, is calculated as $V_{\text {in }} / I_{\text {in }}$ and $S_{11}$ is calculated as $\left(Z_{\text {in }}-Z_{o}\right) /\left(Z_{\text {in }}+Z_{o}\right)$, assuming $Z_{o}=50 \Omega$ for the characteristic impedance of the feedline. Finally, the simulated $S_{11}$ is converted to VSWR. As shown in Fig. 2, both the measured and simulated VSWRs are less than 1.5 over the entire frequency range of interest.

The UWB performance of the antenna can be characterized in the time domain by the fidelity $(F)$, which is a measure of how accurately the transmitted waveform reproduces the time derivative of the voltage applied to the antenna terminals or, equivalently, how accurately the received voltage reproduces the transient field incident upon the antenna [12]. To investigate the antenna's fidelity in transmission and reception of the UWB signal, $e(t)$, shown in Fig. 3(a), two replicas of the antenna shown in Fig. 1 are connected to the two ports of the PNA 


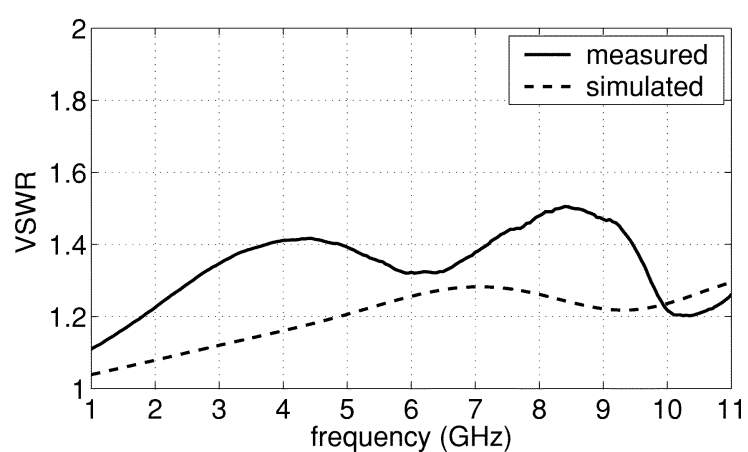

Fig. 2. Simulated and measured VSWR of the antenna.

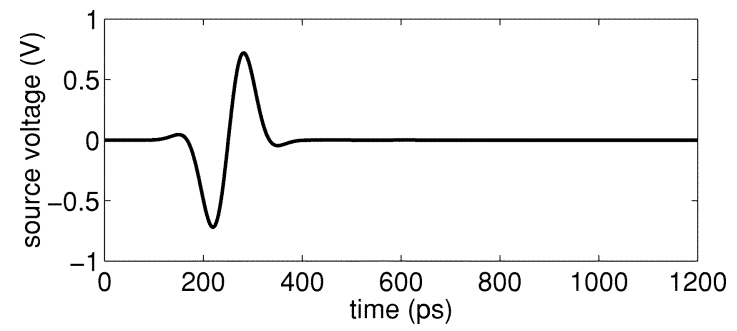

(a)

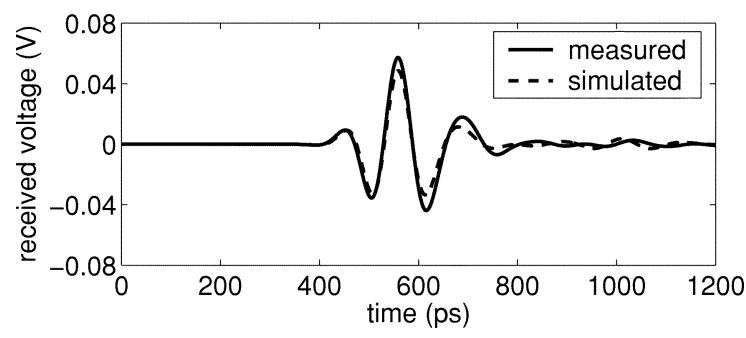

(b)

Fig. 3. (a) Source waveform applied to the input terminals of the transmitting antenna. (b) FDTD-computed and measured waveforms recorded at the receiving antenna located at a distance of $5 \mathrm{~cm}$ from the transmitter.

and aligned face-to-face with a 50 -mm separation between the ends of the pyramidal horns. The forward transmission coefficient $\left(S_{21}\right)$ is measured and converted to a frequency-domain received voltage $V_{2}=S_{21} E / 2$, where $E$ is the spectrum of the desired source waveform $e(t)=e^{-(t-4 \tau)^{2} / \tau^{2}} \sin \left(2 \pi f_{0}(t-\right.$ $4 \tau)$ ) with $f_{0}=6 \mathrm{GHz}$ and $\tau=63 \mathrm{ps}$. Then $V_{2}$ is transformed to the time-domain waveform $v_{2}(t)$ using an inverse DFT. In the FDTD simulation, the two antennas are modeled using a configuration similar to the experimental setup. The source waveform, $e(t)$, is applied at the input terminals of the transmitting antenna and the voltage $v_{2}(t)$ at the receiving antenna is recorded directly. As shown in Fig. 3(b), there is excellent agreement between the simulated and measured versions of $v_{2}(t)$.

The fidelity, defined as $F=\max _{\tau} \int_{-\infty}^{\infty} \hat{r}(t-\tau) \hat{f}(t) d t$, corresponds to the maximum magnitude of the cross correlation between the normalized observed response $\hat{r}(t)$ and the ideal response $\hat{f}(t)$. A fidelity of $F=1$ indicates a perfect match between $\hat{r}(t)$ and $\hat{f}(t)$. Here, $\hat{f}(t)$ is calculated as the normalized time derivative of the source waveform, $s(t)$, and $\hat{r}(t)$ is calculated using the normalized versions of the simulated and measured waveforms plotted in Fig. 3(b). This calculation yields a fidelity of approximately 0.96 in both the simulation and experiment.
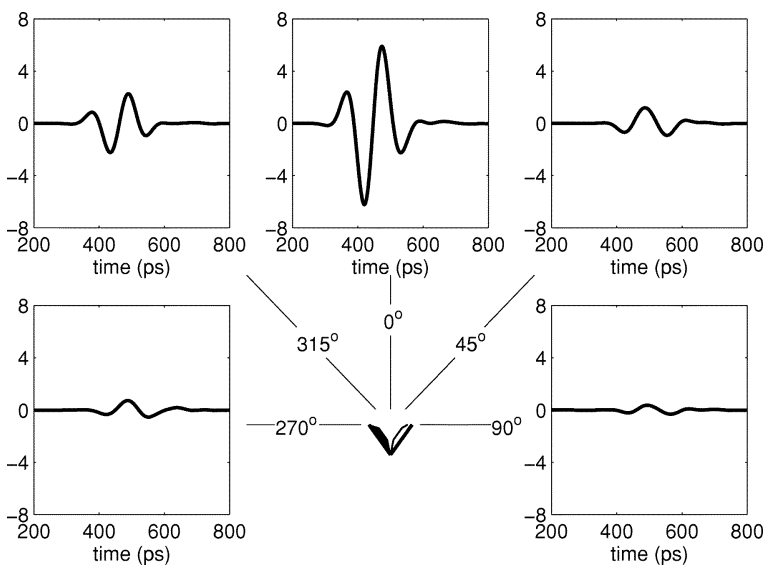

(a)
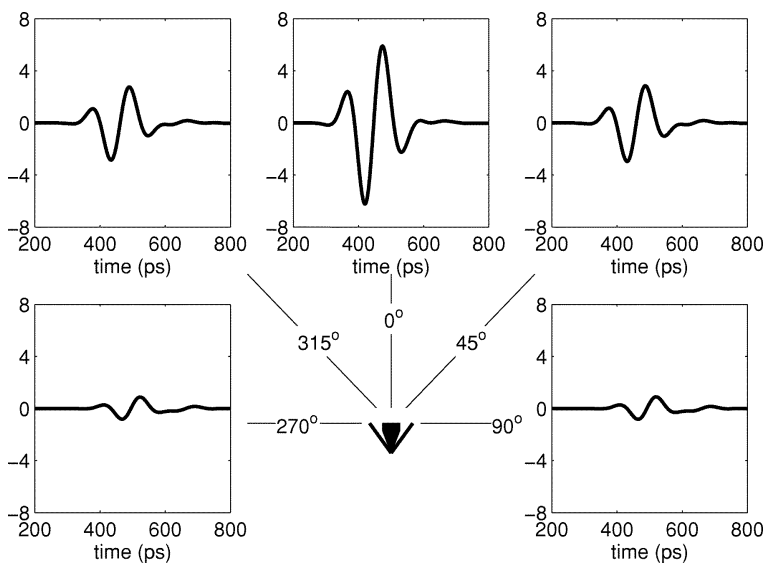

(b)

Fig. 4. Electric-field waveforms computed as a function of observation angle at a constant distance of $5 \mathrm{~cm}$ from the transmitting antenna. (a) $E$-plane waveforms with the launching plane positioned on the right side. (b) $H$-plane waveforms.

To examine the fidelity as a function of angle off boresight, we use FDTD simulations to compute the transient fields radiated by the transmitting antenna at several observation points. Fig. 4(a) shows the radiated electric field waveforms observed at a distance of $50 \mathrm{~mm}$ from the antenna over an angular span of $90^{\circ}$ on either side of boresight in the $E$-plane. Here, the $E$-plane intersects the launching plane and ridge and divides the antenna geometry into two symmetric halves. Fig. 4(b) shows the radiated field in the $H$-plane, which passes between the launching plane and ridge. The fidelity values calculated for all the displayed waveforms are equal to or greater than 0.92 . The waveforms in Fig. 4 also illustrate the antenna's directivity.

Finally, we investigate the directivity in the far field at discrete frequencies. For the radiation pattern measurements, the transmitting and receiving antennas are separated by a distance of $300 \mathrm{~mm}$. The transmitting antenna is rotated in $5^{\circ}$ increments in both the $E$ and $H$ planes. The magnitude of $S_{21}$, which is proportional to the intensity of the field radiated by the transmitter, is recorded at a given frequency as a function of rotation angle. In the FDTD simulation, a sinusoidal waveform with the desired frequency is excited at the feed point and a frequency-domain near-field-to-far-field transformation is used to calculate the far field pattern. Fig. 5 shows good agreement between the simulated and measured antenna radiation patterns 


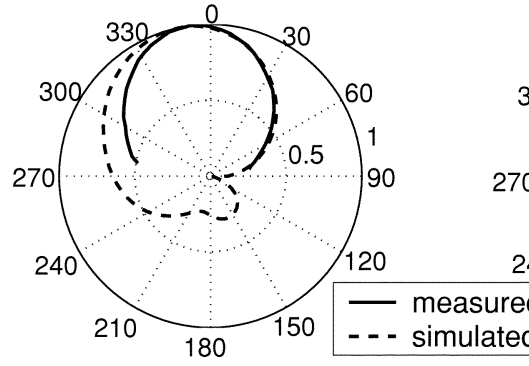

(a)

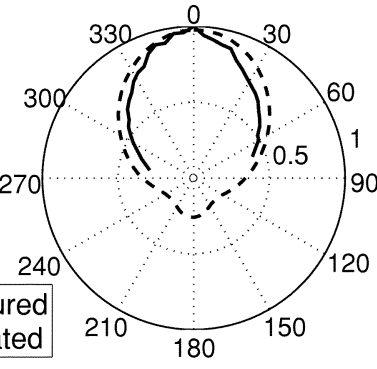

(b)
Fig. 5. Simulated and measured far-field radiation patterns at $6 \mathrm{GHz}$. (a) $E$-plane pattern with the launching plane positioned on the right side. (b) $H$-plane pattern.

at $6 \mathrm{GHz}$. Because of the asymmetry in the antenna geometry, the electric field in the $E$-plane is concentrated in the region between the launching plane and the opposing side wall before being launched to free space. Consequently, the $E$-plane radiation pattern exhibits a slight tilt (approximately $15^{\circ}$ ) toward the ridge. The $H$-plane radiation pattern is symmetric about boresight because of the symmetry exhibited by the antenna in this plane. Simulations and measurements conducted at other frequencies indicate that the directivity increases with frequency and that the far-field radiation patterns exhibit a compact main lobe and minimum sidelobes over the entire frequency range of interest.

We note that the antenna was placed in free space to maintain consistency across all characterizations, including the far-field pattern measurements which are not easily conducted in other media. However, for some UWB radar applications such as space-time microwave imaging for breast cancer detection, the antenna needs to be immersed in a medium other than free space to ensure reasonable coupling of the UWB signals into the biological tissue. We have performed additional experiments and simulations with this type of antenna immersed in a low-loss liquid immersion medium and have achieved results for VSWR and $F$ that are comparable to those reported here.

\section{CONCLUSION}

We have presented a numerical and experimental investigation of a novel UWB antenna-a pyramidal-horn antenna with a single ridge and a curved launching plane. The an- tenna has been designed with centimeter-scale dimensions for low-power UWB microwave radar applications. Both the FDTD simulations and physical measurements indicate that the VSWR is less than 1.5 over the frequency range from 1 to $11 \mathrm{GHz}$. The fidelity ranges between 0.92 and 0.96 over an angular span of $180^{\circ}$ centered on boresight. These results indicate that this antenna offers high-fidelity transmission and reception of ultrashort microwave pulses with minimal distortion. Overall, we conclude that this type of antenna is an excellent candidate for a variety of UWB microwave radar applications, including biological sensing and imaging applications.

\section{REFERENCES}

[1] E. J. Bond, X. Li, S. C. Hagness, and B. D. Van Veen, "Microwave imaging via space-time beamforming for early detection of breast cancer," IEEE Trans. Antennas Propagat., vol. 51, pp. 1690-1705, Aug. 2003.

[2] T. T. Wu and R. W. P. King, "The cylindrical antenna with nonreflecting resistive loading," IEEE Trans. Antennas Propagat., vol. AP-13, pp. 369-373, May 1965.

[3] J. G. Maloney and G. S. Smith, "A study of transient radiation from the Wu-King resistive monopole-FDTD analysis and experimental measurements," IEEE Trans. Antennas Propagat., vol. 41, pp. 668-676, May 1993.

[4] S. C. Hagness, A. Taflove, and J. E. Bridegs, "Three-dimensional FDTD analysis of a pulsed microwave confocal system for breast cancer detection: design of an antenna-array element," IEEE Trans. Antennas Propagat., vol. 47, pp. 783-791, May 1999.

[5] D. W. van der Weide, "Planar antennas for all-electronic terahertz systems," J. Opt. Soc. Amer. B, vol. 11, pp. 2553-2560, 1994.

[6] K. L. Shlager, G. S. Smith, and J. G. Maloney, "Accurate analysis of TEM horn antennas for pulse radiation," IEEE Trans. Electromagn. Compat., vol. 38, pp. 414-423, Aug. 1996.

[7] K. L. Walton and V. C. Sundberg, "Broadband ridged horn design," Microwave J., vol. 4, pp. 96-101, Apr. 1964.

[8] B. M. Notaros, C. D. McCarrick, and D. P. Kasilingam, "Two numerical techniques for analysis of pyramidal horn antennas with continuous metallic ridges," in Proc. IEEE Int. Symp. Antennas Propagation Dig., vol. 2, Boston, MA, 2001, pp. 560-563.

[9] E. T. Rosenbury, G. K. Burke, S. D. Nelson, R. D. Stever, G. K. Gorverno, and D. J. Mullenhoff, "Low Cost Impulse Compatible Wideband Antenna," U.S. Patent 6348898.

[10] M. Piket-May, A. Taflove, and J. Baron, "FDTD modeling of digital signal propagation in 3-D circuits with passive and active loads," IEEE Trans. Microwave Theory Tech., vol. 42, pp. 1514-1523, Aug. 1994.

[11] J. P. Berenger, "A perfectly matched layer for the absorption of electromagnetic waves," J. Comput. Phys., vol. 114, pp. 185-200, 1994.

[12] O. E. Allen, D. A. Hill, and A. R. Ondrejka, "Time-domain antenna characterizations," IEEE Trans. Electromagn. Compat., vol. 35, pp. 339-345, Aug. 1993. 